\title{
Participación de residentes de cirugía plástica de Chile en la publicación científica: Revisión de los últimos 20 años
}

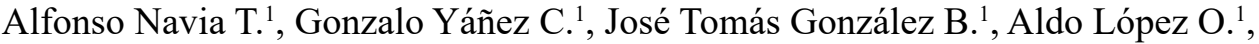 \\ Álvaro Cuadra C. ${ }^{1}$, Bruno Dagnino U. ${ }^{1}$ y Susana Searle F. ${ }^{1}$
}

Participation of chilean plastic surgery residents in scientific publications: Review of the last 20 years

\begin{abstract}
Aim: Evaluate the participation of Chilean plastic surgery residents in scientific publication in the last 20 years and assess their experience during residency. Materials and Method: Literature review from 19982018 under the terms: Cirugia Plastica AND Plastic Surgery AND Chile. Publications with at least one plastic surgeon author with filiation reported in Chile were considered. Those with reported participation of residents were registered and their authors were also analyzed according to their period of residence and date of publication, adding them as unreported residents. Subjects, year of publication and journals were analyzed. A survey was applied to plastic surgery residents and recent plastic surgery graduates to evaluate the perception of their participation in scientific activities. Residents participation with and without a previous research fellow was compared using Fisher's exact test. Results: Reconstructive themed studies $(48.2 \%)$, in adults $(68.6 \%)$ and in university centers $(48.7 \%)$ prevailed among the included articles. The reported participation of residents was $8.4 \%$, which rised to $38.2 \%$ when it was extended to those not explicitly reported as residents among the authors. Residents exposed the lack of time as the main barrier to publication and congress participations. Discussion: Participation in scientific activities is beneficial for residents, their mentors and the academic reputation of their centers. The majority of residents believe that their participation could have been greater if more facilities had been given. Conclusions: Participation of plastic surgery residents in scientific publications is under reported. The implementation of research programs, protected times and active mentoring could increase this number.

Keywords: plastic surgery; bibliometrics; publication; residents; Chile.
\end{abstract}

\section{Resumen}

Objetivo: Analizar la participación de los residentes de cirugía plástica de Chile en la publicación científica de los últimos 20 años y evaluar su experiencia durante la residencia. Materiales y Método: Revisión de la literatura desde 1998-2018 bajo los términos: Cirugía Plástica, Plastic Surgery y Chile. Se incluyeron aquellos con al menos un autor cirujano plástico con filiación en Chile. Se registró la participación reportada de residentes y analizaron sus autores según su período de residencia y fecha de publicación, agregándolos como residentes no reportados. Se analizó tema, año de publicación y revista. Se aplicó una encuesta a residentes de cirugía plástica y postbecados recientes para conocer la percepción sobre su participación en actividades científicas. Se comparó la participación entre residentes con y sin año de investigación mediante el test exacto de Fisher. Resultados: Predominó la temática reconstructiva $(48,2 \%)$, en adultos $(68,6 \%)$ y en centros universitarios $(48,7 \%)$. La participación reportada de residentes fue de $8,4 \%$, subiendo a $38,2 \%$ al ampliarla a los no explicitados como residentes. Los encuestados expusieron la falta de tiempo como principal impedimento a la publicación y participación en congresos. Discusión: La participación en actividades científicas resulta beneficiosa para residentes, sus tutores y la reputación académica de sus centros. La mayoría de los residentes cree que su participación podría haber sido mayor en caso de que se hubiesen dado más facilidades. Conclusiones: La participación de residentes de cirugía plástica se encuentra subreportada. Programas de investigación, tiempos protegidos y mayor tutorización podrían aumentar esta cifra.

Palabras clave: cirugía plástica; bibliometría; publicación; residentes; Chile.
'División de Cirugía, Pontificia Universidad Católica de Chile. Santiago, Chile

Recibido el 15 de agosto de 2019 y aceptado para publicación el 16 de diciembre de 2019.

Correspondencia a: Dr. Alfonso Navia T ajnavia@uc.cl 


\section{Introducción}

La investigación científica es una actividad académica fundamental, permitiendo generar y compartir conocimientos con la comunidad médica. Un porcentaje importante de las publicaciones provienen de centros universitarios, generalmente impulsados por residentes en formación, sin embargo, no existen estadísticas que objetiven y detallen su participación en Chile. Además, con frecuencia se omite explicitar su calidad de residentes dentro de los autores, muchas veces debido a limitaciones en los detalles de filiación de las propias revistas ${ }^{1}$. La literatura avala que la participación de los residentes de cirugía plástica (RCP) en investigación logra desarrollar capacidades de pensamiento crítico y mayor madurez profesional, independiente de si después el residente pretende o no seguir una carrera académica $^{2}$.

Para lograrlo, sin embargo, el residente debe lograr balancear las responsabilidades clínicas con las iniciativas de investigación, contando pocas veces con tiempo protegido para ello. No existe ninguna estadística nacional y hay escasa literatura a nivel internacional sobre la experiencia, barreras y actitudes de los residentes de cirugía plástica hacia la investigación científica ${ }^{3}$.

El objetivo de este trabajo es analizar la participación de los RCP de Chile en la publicación científica nacional e internacional durante los últimos 20 años y evaluar su experiencia durante la residencia en la participación de actividades científicas como publicaciones $\mathrm{y} / \mathrm{o}$ presentaciones orales en congresos.

\section{Materiales y Método}

Se realizó una revisión de la literatura de manera sistemática por 2 autores independientes en Pubmed, Lilacs, Scielo, Central (Cochrane) y Tripdatabase, desde el 1 de enero de 1998 al 1 de junio de 2018 bajo los términos:(Cirugía Plástica OR Plastic Surgery) AND Chile. Se realizó una búsqueda dirigida en la Revista Médica de Chile a través de su portal online (https://scielo.conicyt.cl/rmc.htm) y la Revista de Cirugía (https://scielo.conicyt.cl/rchcir.htm), filtrando los artículos fuera de las fechas incluidas para la revisión. En el caso de Scielo y Lilacs, las búsquedas se hicieron por separado en español (Cirugía Plástica AND Chile) e inglés (Plastic Surgery AND Chile). Se amplió la búsqueda a los portales Epistemonikos y Scopus para aumentar volumen de artículos, también de manera separada en español (Cirugía AND Plástica AND Chile) e inglés (Plas- tic AND Surgery AND Chile). Además, se realizó, una búsqueda dirigida en el portal de la Revista de Cirugía Plástica Ibero-Latinoamericana indexada en Scielo desde 2006 y se buscó manualmente en los archivos descargables de la revista digitalizada desde 2001 hasta 2005 en su página web (https:// ciplaslatin.com). Los criterios de inclusión para el análisis fueron: artículos en los cuales al menos uno de sus autores fuese cirujano plástico o RCP que reportase filiación a un centro en Chile. Se excluyeron publicaciones de centros o equipos de cirugía plástica realizadas por especialistas en otras áreas (traumatología, cirugía oncológica, cirugía maxilofacial, entre otros) que reportaban ser miembros de dichos equipos. Se excluyeron además artículos repetidos en las búsquedas, comentarios de otros artículos, ensayos clínicos o revisiones sistemáticas en desarrollo (no publicados), abstracts o posters de presentaciones en congresos, tesis, blogs y capítulos de libros. Para el análisis de membresía de la Sociedad Chilena de Cirugía Plástica (SCCP) se usó el registro web de su página oficial (http://www. sccp.cl). Dado la gran heterogeneidad en la forma de reportar autoría y filiación de instituciones en las distintas revistas, en caso de no reportarse un centro único o coincidir con el autor de correspondencia, se convino como primera opción establecer el centro indicado por la mayoría de los autores, siempre y cuando incluya al cirujano plástico asignado como primer autor. En caso de ser reportados múltiples centros, se analizó al primer autor que sea cirujano plástico dentro del orden de autores. En caso de que éste a su vez manifieste filiación a múltiples centros, se usó como criterio el último centro reportado por dicho autor. El registro de participación de residentes se hizo en relación al reporte explícito de la calidad de RCP dentro de los autores. Se analizó además a los autores según sus años de residencia y la fecha de publicación del artículo, señalándolos como "Residentes No Reportados" (RNR) cuando corresposponda. La información anterior se obtuvo del Registro de la Superintendencia de Salud del Gobierno de Chile (http://webhosting.superdesalud. gob.cl). Junto con lo anterior se realizó un análisis del tema del estudio, su grupo etario objetivo, el año de publicación y la revista. Todos los datos recopilados fueron revisados por los autores con el fin de asegurar concordancia en el análisis. En los casos de discrepancia, se discutió y llegó a consenso para establecer un registro homogéneo en su criterio.

De forma complementaria, se aplicó una encuesta a los residentes de cirugía plástica y postbecados recientes, considerados aquellos egresados posteriores al año 2015. De los residentes cursando la 
subespecialidad se incluyeron sólo residentes de $1^{\text {er }}$ año que llevaran mínimo 8 meses de su residencia. Se realizó a través de la plataforma Google Forms (https://docs.google.com/forms), contando con 8 preguntas para conocer su percepción acerca de su participación como residentes en actividades científicas. Luego de responder la primera parte, se desplegaba una segunda sección de 3 preguntas para aquellos que contestaban "Sí" a la pregunta: “¿Siente que su participación en congresos y/o publicaciones podría haber sido mayor en caso de que se hubiesen corregido algunos factores o se hubiesen dado más facilidades durante su residencia?". Los datos fueron tabulados usando el programa Microsoft Excel (v. 16.9) y se usó estadística descriptiva de los datos recogidos para representar su tendencia. Se comparó, además, la participación en congresos y publicaciones referida en la encuesta, tanto nacionales como internacionales, por residentes con y sin un año de investigación y se analizó mediante la prueba exacta de Fisher en IBM SPSS v.22. El análisis de los resultados de la encuesta fue realizado de manera anónima.

\section{Resultados}

La búsqueda arrojó un total de 386 artículos. De ellos, tras aplicar criterios de inclusión/exclusión y eliminar aquellos repetidos, quedaron 191 artículos que fueron analizados. Un 97,4\% de los artículos (186) contaban con al menos uno de sus autores como cirujano plástico inscrito en la SCCP. Respecto al grupo etario, la mayoría fueron estudios de adultos (68,6\%), seguido de los pediátricos $(9,9 \%)$ y en tercer lugar los mixtos (adultos más pediátricos) (6,8\%). Un 14,7\% fue catalogado como "otros". Sobre los centros, la mayoría de las filiaciones fueron a universidades u hospitales universitarios $(48,7 \%)$, seguido de mutualidades $(22,5 \%)$, hospitales o instituciones públicas $(11,5 \%)$, hospitales de Fuerzas Armadas (8,4\%) y clínicas privadas (7,9\%). El 1\% fue catalogado como "otros". Predominó como temática la reconstructiva $(48,2 \%)$, luego lo estético $(29,8 \%)$, quemados $(8,4 \%)$, simulación y/o estudios en animales $(3,7 \%)$ y los de biología molecular y células madres e historia de la cirugía plástica, ambos con $2,1 \%$. Un 2,6\% fue catalogado como "Otros". Respecto a la distribución por revista, predominó la "Revista de Cirugía" (33\%), seguida por "Plastic and Reconstructive Surgery" (19,4\%) y "Revista de Cirugía Plástica Ibero-Latinoamericana" (16,2\%) (Figura 1). Al analizar las publicaciones a lo largo del tiempo, vemos una tendencia en aumento desde el año 2005 (Figura 2). Respecto a la participación de residentes, un 8,4\% de los artículos presentaba reporte explícito de autores en calidad de RCP. Al analizar individualmente los autores según sus años de residencia y el momento de publicación del artículo, esta cifra aumentó a un 38,2\% (73), es decir, hubo 57 artículos en los cuales alguno de los autores era RCP y no fue reportado como tal.

En cuanto a los resultados de la encuesta, ésta fue respondida por un total de 25 personas $(89,3 \%$ tasa de respuesta), de los cuales la mayoría eran postbecados recientes de cirugía plástica $(60 \%)$, seguido por un $20 \%$ de residentes de último año $\left(3^{\text {er }}\right.$ año en la Pontificia Universidad Católica de Chile o $2^{\text {do }}$ año

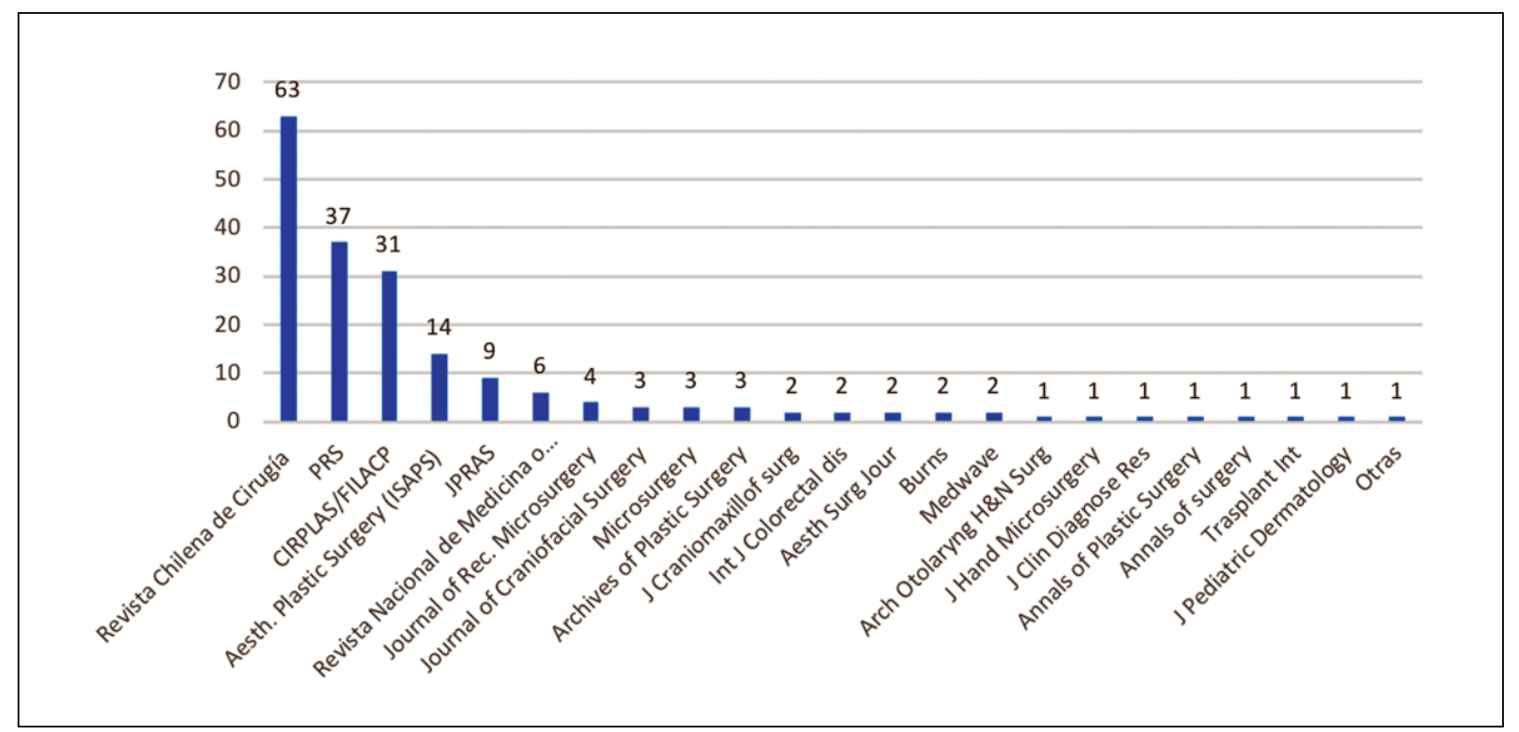

Figura 1. Gráfico con distribución de revistas de las publicaciones de cirujanos plásticos de Chile entre 1998 y 2018. 
en la Universidad de Chile), $12 \%$ residentes de primer año de ambas Universidades y un 4\% residentes de $2^{\text {do }}$ año de la Pontificia Universidad Católica de Chile. Un 32\% (8) reporta haber realizado un año o fellow de investigación en cirugía plástica previo

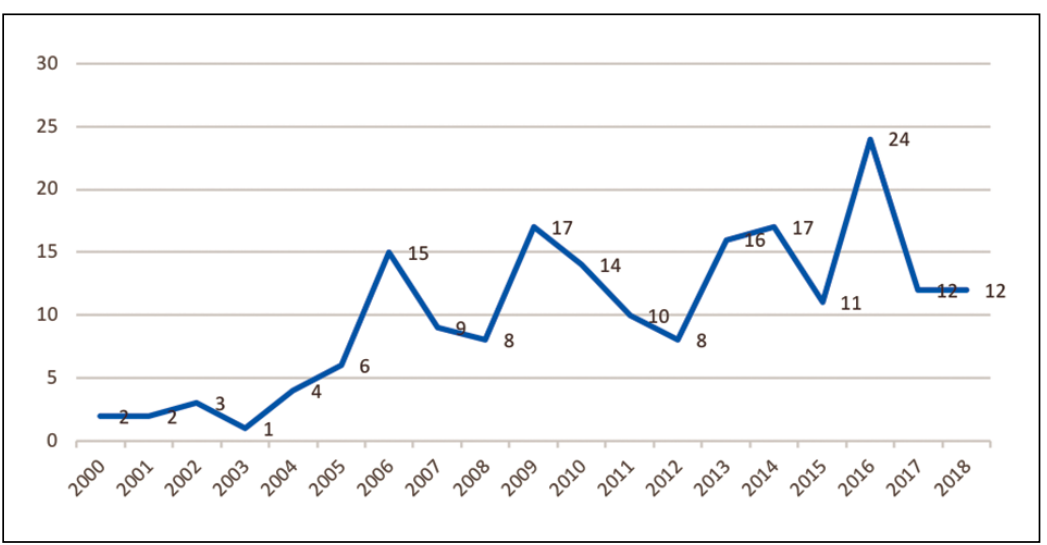

Figura 2. Gráfico con tendencia en el tiempo de las publicaciones de cirujanos plásticos de Chile entre 1998 y 2018. a la residencia clínica. Respecto a la participación en congresos nacionales durante el periodo de residencia, un $92 \%$ de los encuestados refirió haber participado como primer autor, coautor o ambos, en cambio un $48 \%$ reportó participación en congresos internacionales como autor y/o coautor (Figura 3). Sobre la publicación en revistas nacionales, un $60 \%$ refierió haber participado como autor y/o coautor, similar a las revistas internacionales con un $64 \%$ (Figura 4). De los encuestados, un 64\% afirmó haber realizado "todos o la mayoría de los trabajos publicados o presentados en congresos en su centro formador y algunos en rotaciones externas", seguido por la opción "la mayoría en rotaciones externas y algunas en centro formador" con un $24 \%$ y "todas en rotaciones externas" con un $12 \%$. La mayoría de los encuestados (72\%) sintió que su participación en congresos o publicaciones podría haber sido mayor en caso de que se hubieran corregido algunos factores o se hubieran dado más facilidades durante su residencia (Figura 5). Los 18 residentes que respondieron "Sí" a esta última pregunta, atribuyeron
Figura 3. Gráfico A: Participación en congresos nacionales; Gráfico B: Participación en congresos internacionales.
Figura 4. Gráfico A: Participación en publicaciones nacionales; Gráfico B: Participación en publicaciones internacionales.
Participación en Congresos Nacionales

Participación Congresos Internacionales
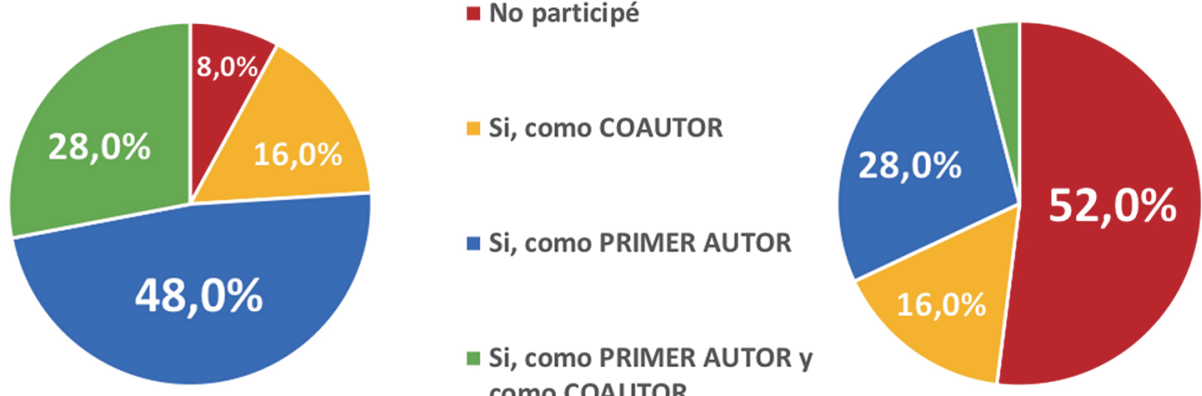

- No participé
— Si, como COAUTOR
" Si, como PRIMER AUTOR
" Si, como PRIMER AUTOR y
como COAUTOR

Publicaciones Revistas Internacionales

Publicaciones Revistas Nacionales

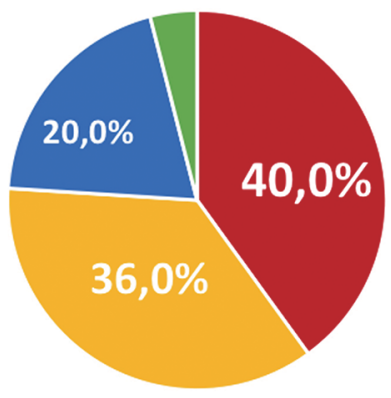

- No participé

- Si, como COAUTOR

E Si, como PRIMER AUTOR

a Si, como PRIMER AUTOR y COMO COAUTOR

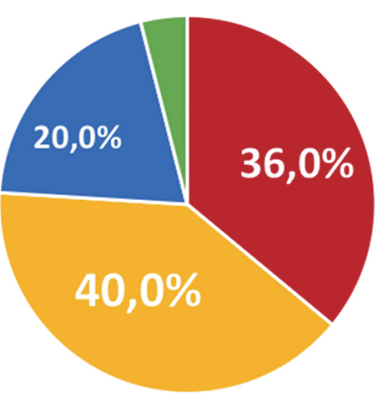


¿Siente que su participación en congresos y/o publicaciones podría haber sido mayor en caso de que se hubiesen corregido algunos factores o se hubiesen dado más facilidades durante su residencia?

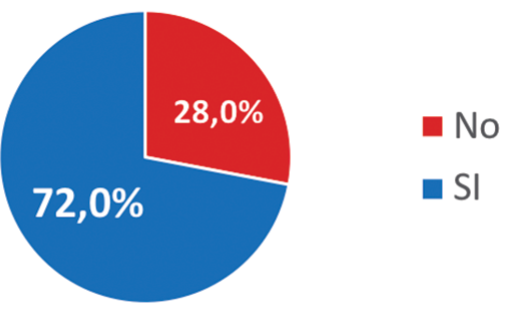

Figura 5. Respuesta a Pregunta: "¿Siente que su participación en congresos y/o publicaciones podría haber sido mayor en caso de que se hubiesen corregido algunos factores o se hubiesen dado más facilidades durante su residencia?". lo anterior a las causas representadas en la Figura 6. La mayoría consideró como el principal factor la falta de tiempo $(66,7 \%)$, seguido por la falta de motivación o tutoría por staff (16,7\%) (Figura 7). De éstos 18 residentes, un 27,8\% reporta haber concretado "más del $50 \%$ de sus ideas para trabajos o publicaciones" durante su residencia, 38,9\% "menos del 50\%" y el restante 33,3\% "pocas veces logró concretar" sus ideas de investigación. Ningún encuestado refirió que pudo presentar o publicar el $100 \%$ de los proyectos de trabajos ideados durante su residencia.

Al analizar a aquellos residentes que habían realizado un año previo o fellow de investigación en cirugía plástica, podemos ver que la principal diferencia fue en relación a las publicaciones en revistas internacionales con un $100 \%$ de participación en los residentes de cirugía plástica con 1 año de investigación en comparación con un $47,1 \%$ de
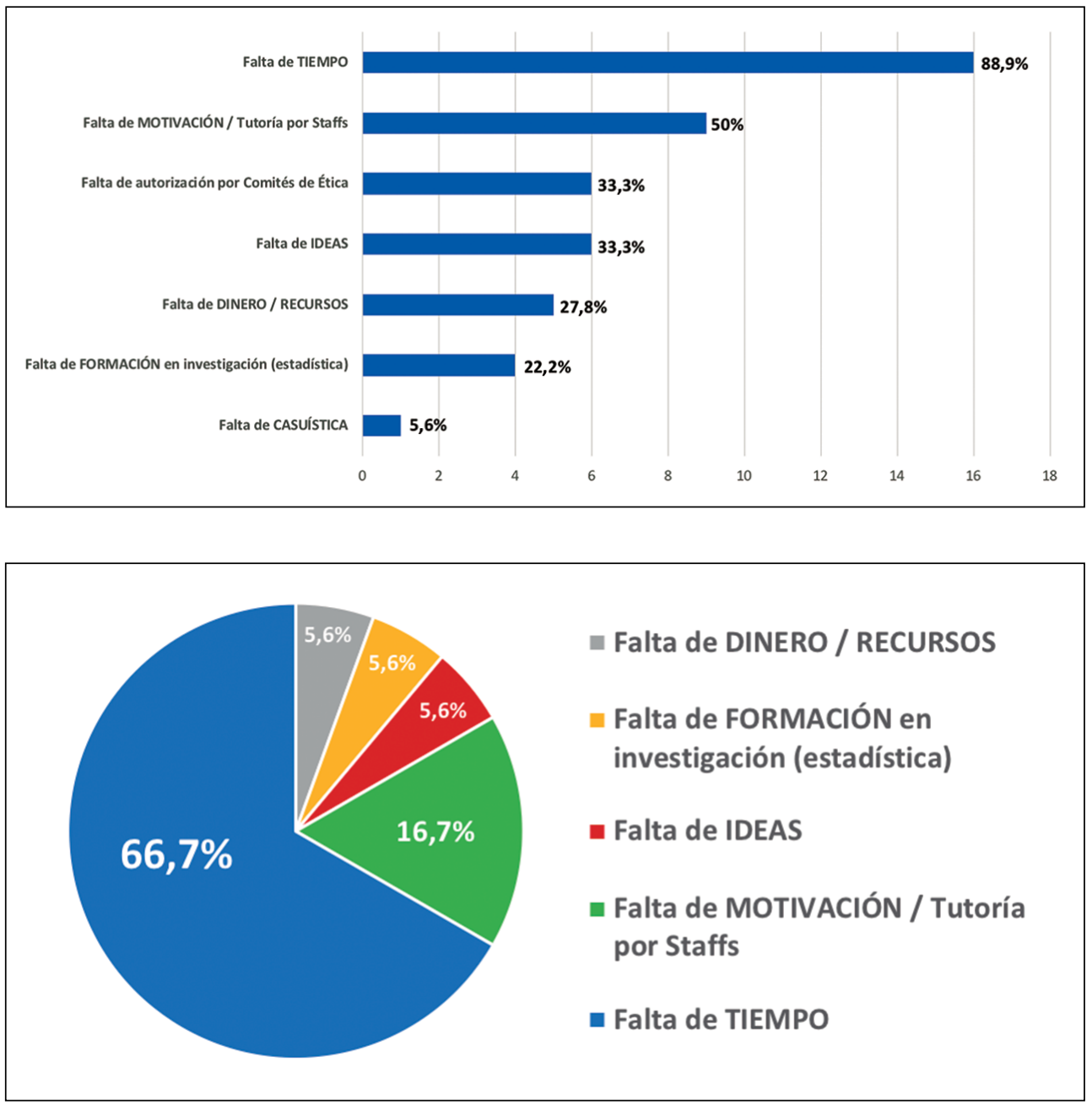

Figura 6. Respuesta a Pregunta: "Razones para la falta de involucramiento de RCP en actividades de investigación y congresos... (marque todas aquellas que lo identifiquen)".
Figura 7. Respuesta a Pregunta: "De todas las razones anteriores, ¿cuál cree que fue la PRINCIPAL RAZÓN por la cual no participó tanto en congresos/publicaciones?". 
Tabla 1. Comparación de resultados de encuesta entre residentes con y sin un año (fellow) previo de investigación en cirugía plástica

\begin{tabular}{|c|c|c|}
\hline & $\begin{array}{l}\text { CON } 1 \text { año de investigación } \\
\text { en cirugía plástica }(n=8)\end{array}$ & $\begin{array}{l}\text { SIN } 1 \text { año de investigación en cirugía plástica } \\
(n=17)\end{array}$ \\
\hline $\begin{array}{l}\text { Presentaciones congresos nacionales* } \\
(\mathrm{p}=1,0)^{* *}\end{array}$ & $\begin{array}{cl}100 \% & \text { SÍ } \\
0 \% & \text { NO }\end{array}$ & $\begin{array}{ll}89,2 \% & \text { SÍ } \\
11,8 \% & \text { NO }\end{array}$ \\
\hline $\begin{array}{l}\text { Presentaciones congresos internacionales* } \\
(p=0,4)^{* *}\end{array}$ & $\begin{array}{ll}62,5 \% & \text { SÍ } \\
37,5 \% & \text { NO }\end{array}$ & $\begin{array}{ll}41,2 \% & \text { SÍ } \\
58,8 \% & \text { NO }\end{array}$ \\
\hline $\begin{array}{l}\text { Publicaciones revistas nacionales* } \\
(\mathrm{p}=0,088)^{* *}\end{array}$ & $\begin{array}{ll}87,5 \% & \text { SÍ } \\
12,5 \% & \text { NO }\end{array}$ & $\begin{array}{ll}47,1 \% & \text { SÍ } \\
52,9 \% & \text { NO }\end{array}$ \\
\hline $\begin{array}{l}\text { Publicaciones revistas internacionales* } \\
(\mathrm{p}=0,022)^{* *}\end{array}$ & $\begin{aligned} 100 \% & \text { SÍ } \\
0 \% & \text { NO }\end{aligned}$ & $\begin{array}{ll}47,1 \% & \text { SÍ } \\
52,9 \% & \text { NO }\end{array}$ \\
\hline $\begin{array}{l}\text { ¿Su participación en investigación pudo haber } \\
\text { sido mayor? }\end{array}$ & $\begin{array}{ll}50 \% & \text { SÍ } \\
50 \% & \text { NO }\end{array}$ & $\begin{array}{ll}82,3 \% & \text { SÍ } \\
17,6 \% & \text { NO }\end{array}$ \\
\hline $\begin{array}{l}\text { ¿Cuál cree fue la principal razón por la cual no } \\
\text { participó tanto? }\end{array}$ & $100 \%$ falta de TIEMPO & $\begin{aligned} 57 \% & \text { falta de TIEMPO } \\
21,4 \% & \text { falta de MOTIVACIÓN o tutoría por Staffs } \\
7,2 \% & \text { falta de DINERO } \\
7,2 \% & \text { falta de IDEAS } \\
7,2 \% & \text { falta de formación en ESTADÍSTICA }\end{aligned}$ \\
\hline $\begin{array}{l}\text { ¿Qué porcentaje de sus proyectos de } \\
\text { investigación logró concretar? }\end{array}$ & $\begin{array}{ll}50 \% & \text { más del } 50 \% \\
50 \% & \text { menos del } 50 \%\end{array}$ & $\begin{array}{ll}21,4 \% & \text { más del } 50 \% \\
35,7 \% & \text { menos del } 50 \% \\
42,9 \% & \text { pocas veces logró concretar }\end{array}$ \\
\hline
\end{tabular}

*Participación como autor y/o coautor ${ }^{* *}$ Se consideró estadísticamente significatico $\mathrm{p}=<0,05$.

aquellos que no lo realizaron, lo cual fue estadísticamente significativo $(\mathrm{p}<0,05)$. Además, en el caso del primer grupo, un $50 \%$ sintió que su participación en congresos $\mathrm{y} / \mathrm{o}$ publicaciones podría haber sido mayor comparado con un $82,3 \%$ de aquellos que no realizaron 1 año de investigación previo a su residencia clínica (Tabla 1 ).

\section{Discusión}

Presentamos el primer trabajo en la literatura nacional que reporta la participación de RCP en investigación científica con una búsqueda y análisis retrospectivo a lo largo de 20 años. Además, incluimos una encuesta realizada a RCP y a cirujanos plásticos recién egresados, logrando conocer información valiosa que permita crear conciencia y optimizar su participación en investigación científica durante la residencia, lo que resultaría en un beneficio tanto para los residentes como sus tutores y la reputación académica de sus programas y centros formadores ${ }^{4}$. El período de residencia es un tiempo altamente exigente tanto en lo teórico como lo asistencial por lo que la inquietud científica de los residentes consideramos debe reconocerse como un mérito adicional.
Un dato relevante es la participación subreportada de residentes $(8,4 \%)$, la cual aumenta a un $38,2 \%$ tras nuestro análisis, sin embargo, lo anterior no es sólo por falta de los autores al momento de detallar la filiación de los mismos, sino que muchas veces producto de restricciones de parte de las revistas. Consideramos relevante detallar la calidad de residentes de sus autores dado que creemos agrega un valor adicional tanto al residente como al artículo. Por otra parte, consideramos aún baja una participación de 38,2\% de residentes, especialmente teniendo en cuenta que un $48,7 \%$ de las publicaciones fue realizada en centros universitarios. Al analizar la encuesta, vemos que la mayoría de los residentes piensa que su participación en congresos y/o publicaciones podría haber sido mayor en caso de que se hubiesen dado más facilidades. Esto, permite concluir que sí existe en la mayoría de los residentes encuestados un verdadero interés de participación, pero por la carga laboral que tienen esto se hace inviable la mayoría de las veces. La razón más frecuentemente referida por los residentes fue la falta de tiempo $(88,9 \%)$ seguido por la falta de motivación y/o tutoría por staff (50\%). Un estudio realizado en Canadá a residentes de 13 programas de formación de cirugía plástica $(n=95)$ concluyó que 
la principal barrera reportada para la investigación fue la falta de tiempo (83\%), seguido de la falta de mentorización/supervisión (42\%), siendo concordantes con nuestros resultados ${ }^{3}$. Otras publicaciones internacionales en residentes de otras especialidades apoyan lo mismo ${ }^{5-7}$. Por ello, es importante incentivar a los centros formadores a crear instancias de formación en investigación, dar tiempo protegido, tutorización activa por parte de sus mentores y desarrollar instancias frecuentes de intercambio científico. La literatura avala que una formación proactiva del punto de vista científico con un mentor a cargo puede incentivar a los residentes a seguir una carrera académica, además de una mayor productividad científica ${ }^{8,9}$. La creación de sistemas de tutorización entre residentes de último año con residentes de primer año también es un método que podría ayudar a motivar y optimizar la productividad científica ${ }^{10}$. Estudios revelan que la realización de reuniones trimestrales de los departamentos de cirugía plástica aumentarían de manera significativa la tasa de presentaciones en congresos nacionales y regionales y la tasa de publicaciones aumentarían en hasta 4 veces ${ }^{4}$.

La investigación científica en RCP es un componente crítico durante la residencia. Esto incluye varios beneficios, destacando en la literatura que el residente desarrolle una visión más crítica, relaciones más fuertes con sus mentores y contribuya a la cirugía plástica del futuro con innovación y nuevos descubrimientos científicos ${ }^{2,8}$. La presentación en cursos y congresos no solo le da al residente la posibilidad de exponerse y hacer redes de contacto, sino también ganar reconocimiento y seguridad en la productividad académica.

El mejor ejemplo para la optimización de la producción científica es la presencia de residentes de investigación, quienes durante 1 año con dedicación exclusiva al trabajo no clínico, potencian la productividad científica del equipo, institución y sus pares residentes cursando la etapa clínica, permitiendo liderar los proyectos desde su ideación hasta su publicación. La literatura internacional apoya la existencia de residentes o fellows de investigación en cirugía plástica y promueve la creación de espacios protegidos de tiempo, además, de reuniones periódicas, tutorización adecuada y financiamiento ${ }^{11,12}$. En nuestra experiencia, vimos una mayor participación de los RCP con 1 año previo de investigación tanto en presentaciones en congresos nacionales e internacionales, como publicaciones nacionales e internacionales, diferencia que fue estadísticamente significativa en este último aspecto $(47,1 \%$ vs $100 \%)$.
Este estudio incluye a residentes y postbecados recientes de las únicos dos programas de formación en cirugía plástica en Chile, con un total anual de apenas 5 vacantes entre ambas universidades aproximadamente. Por ende, a pesar de que el número de encuestados es relativamente bajo, nuestra tasa de respuesta fue de casi un $90 \%$ y creemos que nuestros resultados son bastante representativos de la realidad nacional de los últimos 5 años. Un potencial sesgo de selección de nuestro artículo es el hecho de que dado que el objetivo de la encuesta era analizar la participación en actividades científicas, aquellos residentes que no contestaron la encuesta puede que cuenten con una menor participación que los que sí se interesaron en hacerlo.

Además, cabe destacar que por lo general el proceso de publicación desde la generación de la idea hasta su posterior publicación suele demorar al menos 1 año, por lo que puede que existan proyectos en desarrollo que no hayan alcanzado a publicarse durante el período de residencia de 2 o 3 años.

\section{Conclusión}

La participación de los RCP de Chile se encuentra subreportada en la literatura. La principal razón por la cual los residentes refirieron una participación menor a la deseada en investigación fue la falta de tiempo. La existencia de residentes de investigación en cirugía plástica, la creación de espacios protegidos y tutorización activa por parte de sus mentores podría contribuir a optimizar su participación y aumentar esta cifra.

\section{Agradecimientos}

En el desarrollo de este artículo participaron dos residentes de cirugía plástica (A.N y A.L), un residente de cirugía general (G.Y) y un interno de medicina (J.G).

\section{Responsabilidades éticas}

Protección de personas y animales. Los autores declaran que para esta investigación no se han realizado experimentos en seres humanos ni en animales.

Confidencialidad de los datos. Los autores declaran que en este artículo no aparecen datos de pacientes.

Conflictos de interés: no hay. 


\section{Bibliografía}

1. Navia A, López A, González JT, Yáñez G, Searle S. Tendencias y perfil de publicación de los cirujanos plásticos de los 22 países miembros de la FILACP, revisión de los últimos 20 años. Cirugía Plástica Ibero-Latinoamericana. 2019;45:81-9.

2. Ballard TNS, Sando IC, Kasten SJ, Cederna PS. Successfully integrating research into plastic surgery training programs. J Craniofac Surg. 2015;26:2279-82.

3. Al-Taha M, Al Youha S, Al-halabi B, Stone J, Retrouvey H, Samargandi $\mathrm{O}$, et al. Barriers and Attitudes to Research Among Residents in Plastic and Reconstructive Surgery: A National Multicenter Cross-Sectional Study. J Surg Educ. 2017;74:1094-104.

4. Holoyda K, Donato D, Veith J, Simpson
A, Moores N, Agarwal J. A Dedicated Quarterly Research Meeting Increases Resident Research Productivity. J Surg Res [Internet]. 2019;241:103-6. Available from: https://doi.org/10.1016/j. jss.2019.02.041

5. Silcox LC, Ashbury TL, VanDenKerkhof EG, Milne B. Residents' and program directors' attitudes toward research during anesthesiology training: A Canadian perspective. Anesth Analg. 2006;102:85964.

6. Mansi A, Karam WN, Chaaban MR. Attitudes of Residents and Program Directors Towards Research in Otolaryngology Residency. Ann Otol Rhinol Laryngol. 2019;128:28-35.

7. Bammeke F, Liddy C, Hogel M, Archibald D, Chaar Z, MacLaren R. Family medicine residents' barriers to conducting scholarly work. Can Fam Physician. 2015;61:780-7.
8. Mandel BA, Weber SM, Gutowski KA, Neil Salyapongse A, Bentz ML. What influences a plastic surgery resident to pursue an academic career? Plast Reconstr Surg - Glob Open. 2018;6:1-5.

9. Franzblau LE, Kotsis S V., Chung KC. Mentorship: Concepts and application to plastic surgery training programs. Plast Reconstr Surg. 2013;131:837-43.

10. Chetta MD, Sugg KB, Díaz-García RJ, Kasten SJ. Factors influencing american plastic surgery residents toward an academic career. Plast Surg. 2017;25:33-9.

11. Rohrich R, Robinson JJ, Adams WJ. The plastic surgery research fellow: revitalizing an important asset. Plast Reconstr Surg. 1998;102:895-8.

12. Carney MJ, Weissler JM, Koltz PF, Fischer JP, Wu LC, Serletti JM. Academic Productivity, Knowledge, and Education in Plastic Surgery. Plast Reconstr Surg. 2017;140:842-9. 